Forum 2017 · 32:347

DOI 10.1007/s12312-017-0308-9

Online publiziert: 28. Juli 2017

○) Springer Medizin Verlag GmbH 2017

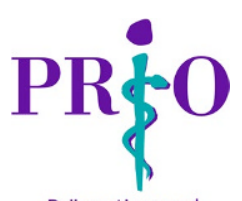

Prävention und

integrative Onkologie

Arbeitsgemeinschaft Prävention und Integrative Onkologie (PRIO)

\title{
Kommunikation in der Onkologie - Teil II der Seminarreihe
}

Einen kurzen Ausflug unternahmen

Der 2. Teil der Seminarreihe fand vom 12.-13.05.2017 in Berlin statt. Während im ersten Teil die Patienten-Arzt-Kommunikation im Vordergrund stand, rückte diesmal die Kommunikation der Professionellen untereinander in den Vordergrund. Stationsvisite, Tumorkonferenz, Fallbesprechung aber auch Mitarbeitergespräch und Change Management Situationen wurden in kleinen Gruppen analysiert und im Hinblick auf ihre Bedeutung für eine patientenzentrierte Medizin, die auch den Ärzten und Pflegekräften das Gefühl gibt, trotz aller Einengungen im Gesundheitssystem eine gute, erfüllende Arbeit zu machen.

Die Organisation lag wieder in den bewährten Händen der „Tagungsschmiede“, dem Veranstaltungsbetrieb der Stiftung Deutsche Leukämie- \& LymphomHilfe.

Auch wenn es um uns „Profis“ ging die Vertreter der Selbsthilfe waren wie im ersten Seminar mit hohem Engagement dabei und brachten aus ihren sehr unterschiedlichen Perspektiven und Erfahrungen viele Anregungen ein. die Teilnehmer jeweils in die Frage der Patienteninformation via Emails bzw. in die Welt der Patientenforen und -chats.

\section{Kommunikationsform: Patiententag}

Als besondere Form der Kommunikation mit Patienten wurde über Patiententage und -vorträge diskutiert. Aus Patientensicht sind diese Veranstaltungen wichtig und die Teilnehmer erhielten aus erster Hand viele Anregungen für die Gestaltung eines solchen Tages in ihren Einrichtungen. Von Ansprache der Selbsthilfe im Vorfeld, Auswahl der Programme zu zeitliche und räumlicher Gestaltung und Bewerbung konnte jeder eine Baukasten an Ideen mit nach Hause nehmen. Und damit am nächsten Patiententag auch die Vorträge „stimmen“ wurde im Plenum und in Kleingruppen erarbeitet, was einen guten Patientenvortrag ausmacht.

Mit diesem Thema wird der dritte Teil im Oktober beginnen: die Teilnehmer werden einen kurzen Patientenvortrag mit einem Thema eigener Wahl vorbereiten und dann im Seminar vorstellen.

Danach wird es wieder um das Patienten-Arzt-Gespräch gehen. Diesmal wird der Fokus auf besonderen Gesprächssituationen liegen. Wir freuen uns erneut auf einen intensiven Gedankenaustausch mit den Patientenvertretern und auf kleine Übungsgruppen, in denen Zeit und Raum ist, um auch schwierige Situationen ebenso wie ungewöhnliche Ideen $\mathrm{zu}$ diskutieren.

\section{Ankündigung}

Das 3. Seminar findet in Berlin am 13.14.10.2017 statt. Dieses Seminar ist auch offen für Teilnehmer, die an den ersten beiden Seminaren nicht teilgenommen haben.

Anmeldungen unter:

Stiftung Deutsche Leukämie- \&

Lymphom-Hilfe

Tel.: 022833889215

Prio-fachtagung@dlh-stiftung.de www.prio-dkg.de 